\title{
Tabularia
}

\section{Les sources de l'histoire du duché. Publications et inventaires récents}

The sources of the history of the duchy. Recent publications and inventories

\section{Pierre Bauduin}

\section{(2) OpenEdition}

\section{Journals}

\section{Édition électronique}

URL : http://journals.openedition.org/tabularia/1860

DOI : 10.4000/tabularia.1860

ISSN : 1630-7364

Éditeur :

CRAHAM - Centre Michel de Boüard, Presses universitaires de Caen

\section{Référence électronique}

Pierre Bauduin, « Les sources de l'histoire du duché. Publications et inventaires récents », Tabularia [En ligne], Guillaume de Volpiano : Fécamp et l'histoire normande, mis en ligne le 12 mars 2003, consulté le 01 mai 2019. URL : http://journals.openedition.org/tabularia/1860; DOI : 10.4000/tabularia.1860 


\title{
Les sources de l'histoire du duché. Publications et inventaires récents.
}

\section{The sources of the history of the duchy. Recent publications and inventories}

\author{
Pierre BAUDUIN \\ CRAHM-UMR 6577, \\ Université de Caen Basse-Normandie \\ crahm.editextes@unicaen.fr
}

Résumé :

Les travaux récents sur l'histoire du duché se sont largement appuyés sur le regard nouveau porté sur les sources mises à la disposition des chercheurs. L'effort de publication des textes narratifs ne s'est pas uniquement traduit par la réédition des ouvres majeures des chroniqueurs normands. Il a aussi conduit à une redécouverte de la première historiographie normande et alimenté les débats portant sur les processus de transmission de la mémoire et l'appropriation, par une société, de son passé.

L'examen de cette production doit prendre en considération les autres témoignages sur l'histoire du duché. Bien qu'il subsiste de nombreux actes inédits, le corpus des textes diplomatiques publiés s'est enrichi et des travaux récents ont inséré le dossier documentaire normand dans le vaste débat sur la «mutation documentaire» du XIe siècle. Egalement sollicitées, les « archives du sol» ont enfin rappelé l'intérêt d'un rapprochement de toutes les données fournies par les textes et l'archéologie pour appréhender l'évolution de la société normande.

Mots-clés : historiographie, hagiographie, diplomatique, chartes, archéologie.

Abstract:

Recent studies on the history of the duchy have largely drawn on the new light shed on sources available to scholars. The endeavours to publish the narrative texts have materialised not only into new editions of the major works of the Norman chroniclers, but have also led to a rediscovery of the earliest Norman historiography and sustained debates concerning the processes of transmission of memory and the appropriation by a society of its past.

The analysis of this production must take into consideration the other accounts of the history of the duchy. Although many acts remain unpublished, the corpus of diplomatic texts has grown and recent studies have inserted the Norman documentary evidence into the large debate on the "documentary transformation" which took place in the $11^{\text {th }}$ century. Finally, the "underground archives", which were also resorted to, have drawn our attention to the importance of bringing together all the data provided by the texts and by archaeological evidence in order to seize the evolution of Norman society.

Keywords: historiography, hagiography, diplomatic, charters, archaeology. 
Le millénaire de la réforme de Guillaume de Volpiano offre l'occasion de rappeler la place tenue par Fécamp dans l'élaboration des matériaux qui permettent de saisir l'histoire du duché à un moment où celui-ci aborde une étape capitale de son évolution. Le foyer fécampois illustre à plus d'un titre le renouveau de la production écrite à l'orée du XI siècle. L'une des expressions de la réapparition de l'écrit documentaire réside dans la charte de fondation de la collégiale de Fécamp en 990. Il n'est pas totalement inutile de rappeler aussi que le plus ancien diplôme ducal connu en original est un acte de Richard II en faveur de l'abbaye de Fécamp daté du 30 mai 1006 et conservé au Musée de la Bénédictine ${ }^{1}$. Entre temps, dès la fin du $\mathrm{X}^{\mathrm{e}}$ siècle, les bases d'une tradition historiographique appelée à durer paraissent avoir été solidement établies à Fécamp. Le site même du palais ducal, enfin, a été l'un des terrains de rencontres privilégiés entre les données fournies par les textes et l'archéologie. S'il n'est pas le seul sollicité, le dossier documentaire fécampois offre ainsi matière à débats sur les sources de la Normandie ducale. Nous privilégierons trois aspects, en tentant de rendre compte des acquis de la recherche, d'abord dans les domaines de l'historiographie médiévale et de la production diplomatique, puis en abordant la diversification des sources à la disposition des historiens de la Normandie. Nous y associerons un corpus de références bibliographiques qui, sans prétendre à l'exhaustivité, tentera de faire le point sur les publications récentes (depuis 1990) de sources ou d'études sur la documentation normande des X ${ }^{e}$-XIII ${ }^{e}$ siècles. Un premier panorama est facilement accessible dans:

La Normandie vers l'an mil, François de BeAurepaire et Jean-Pierre Chaline (éd.), Rouen, Société de l'Histoire de Normandie, 2000, 222 p.

VAN HouTs, Elisabeth M. C., The Normans in Europe, Manchester, Manchester University Press, 2000, 308 p.

\section{La première historiographie du duché redécouverte}

L'une des voies les plus fécondes de la recherche récente a sans doute été une réévaluation de l'historiographie normande à l'époque ducale. Celle-ci a suivi des cheminements très divers, qui reflètent la richesse des perspectives abordées par les spécialistes. La fidélité aux règles les plus scrupuleuses de l'érudition indispensable à l'établissement des textes a ici rencontré l'enrichissement d'un questionnement destiné à comprendre les mécanismes de production et de transmission de la mémoire, à scruter la manière dont une société s'approprie son passé. Les synthèses récentes de Leah Shopkow, d'Elisabeth Van Houts et d'Emily Albu illustrent chacune à leur manière l'élargissement du questionnaire appliqué aux sources en s'interro-

1. Fécamp, Musée de la Bénédictine, $\mathrm{n}^{\circ} 1$ bis, éd. Fauroux, Marie, Recueil des actes des ducs de Normandie (911-1066), Caen (Mémoires de la Société des Antiquaires de Normandie, t. XXXVI), 1961, nº 9 , p. 79-91. Pour les références aux publications plus récentes (depuis 1990), nous renvoyons à la bibliographie dans le cours de cette contribution. 
geant sur les fonctions et les significations de l'histoire dans la Normandie des $\mathrm{XI}^{\mathrm{e}}$ XII ${ }^{\text {e }}$ siècles ou en explorant le rôle tenu par les femmes, au côté des hommes, dans la transmission des faits du passé.

ALBU, Emily, The Normans in their Histories: Propaganda, Myth and Subversion, Woodbridge, Boydell Press, 2001, 260 p.

SHOPKOW, Leah, History and Community: Norman Writing in the Eleventh and Twelfth Centuries, Washington D. C., Catholic University of America Press, 1997, 327 p.

VAN HouTs, Elisabeth M. C., Memory and Gender in Medieval Europe 900-1200, Basingstoke-Londres, Macmillan Press, 1999, 196 p.

VAN HOUTS, Elisabeth M. C., History and Family Traditions in England and the Continent, 1000-1200, Aldershot-Brookfield, Ashgate (Variorum Collected Studies Series), $1999,356 \mathrm{p}$.

Cet élargissement des horizons de la recherche historiographique a d'abord profité aux grandes compositions laissées par les chroniqueurs du duché. La réédition des textes fondamentaux, entamée voici plus d'une trentaine d'années avec la publication de l'Histoire ecclésiastique d'Orderic Vital ${ }^{2}$, s'est poursuivie au cours de la dernière décennie avec la réédition des Gesta Normannorum Ducum de Guillaume de Jumièges, Orderic Vital et Robert de Torigni, des Gesta Guillelmi de Guillaume de Poitiers et du Carmen de Hastingae Proelio de Guy d'Amiens. Il faut y ajouter les nouvelles éditions critiques (et/ou traductions) des chroniqueurs français (Adémar de Chabannes, Raoul Glaber), anglais (Guillaume de Malmesbury, Henri de Huntingdon, Jean de Worcester) ou italiens (Geoffroi de Malaterra) qui intègrent dans leur texte des informations sur l'histoire du duché ou de ses habitants.

Sources éditées, publiées et/ou traduites:

Adémar DE Chabannes, Ademari Cabannensis Chronicon, éd. Pascale BouRgain, Turnhout, Brepols (Corpus Christianorum, Continuatio Mediaeualis, CXXIX), 1999, CXVI +392 p.

The Carmen de Hastingae Proelio of Guy Bishop of Amiens, éd. Frank BARLOw, Oxford, Clarendon Press (Oxford Medieval Texts), 1999, XCI + 55 p.

The Gesta Normannorum Ducum of William of Jumièges, Orderic Vitalis and Robert of Torigni, éd. et trad. Elisabeth M. C. VAN HouTs, Oxford, Clarendon Press (Oxford Medieval Texts), 1992-1995, 2 vol., CXXXIII + 156 p. et XV + 341 p.

Encomium Emmae Reginae, éd. Alistair CAMPBELL, Londres, Camden third series, vol. LXXII, 1949, réimpr. avec introduction de Simon KeYNES, Cambridge, Cambridge University Press, 1998, CLI + 112 p.

Étude critique avec traduction et commentaire des deux premiers livres de la chronique de Geoffroi MAlaterRA, De rebus gestis Rogerii Calabriae e Siciliae comitis et Roberti

2. The Ecclesiastical History of Orderic Vitalis, éd. et trad. M. CHIBNALL, Oxford, Clarendon Press (Oxford Medieval Texts), 1969-1980, 6 vol. 
Guiscardi ducis fratris eius, éd. Maris-Agnès LuCAS-AvENEL, Thèse de doctorat de Langues et littératures anciennes, Universités de Caen Basse-Normandie/Palerme, 2001, 4 vol. en 603 p. (dactyl.).

Guillaume de Malmesbury, Gesta Regum Anglorum, éd. et trad. Roger A. B. MYNORS, Rodney M. Thomson, Michael Winterbottom, Oxford, Clarendon Press (Oxford Medieval Texts), 1998-1999, 2 vol., XXXIII + 879 p. et XLVII + 496 p.

Guillaume de MALmesbury, Historia novella, The contemporary history, éd. Edmund KING, trad. K.R. POTTER, Oxford, Clarendon Press (Oxford Medieval Texts), 1998, $143 \mathrm{p}$.

The Gesta Guillelmi of William of Poitiers, éd. et trad. anglaise, Ralph H. C. DAvis et Marjorie Chibnall, Oxford, Clarendon Press (Oxford Medieval Texts), 1998, XLVII $+199 \mathrm{p}$.

Henri de Huntingdon, Historia Anglorum (History of the English People), éd. et trad. Diana Greenway, Oxford, Clarendon Press (Oxford Medieval Texts), 1996, 899 p.

Jean de WORCESTER: The Chronicle of John of Worcester, II, The Annals from 450 to 1066, éd. et trad. Reginald Ralph DARLINGTON et Patrick MCGURK, III, The Annals from 1067 to 1140 with the Gloucester Interpolations and the Continuation to 1141, éd. et trad. Patrick MCGurK, Oxford, Clarendon Press (Oxford medieval Texts), 19951998, LXXXVI + 717 p. et L + 351 p.

Raoul Glaber, Histoires, traduites et présentées par Mathieu ARnoux, Turnhout, Brepols, 1996, 323 p.

Études:

BouET, Pierre, «L'image des évêques normands dans l'œuvre d'Orderic Vital », Les évêques normands du XI e siècle, Actes du colloque de Cerisy-la-Salle (30 sept.-3 oct. 1993), Pierre Bouet et François Neveux (dir.), Caen, Presses Universitaires de Caen, 1995, p. 253-275.

ChIBnALl, Marjorie, «A Twelfth-Century View of the Historical Church: Orderic Vitalis ", in The Church Retrospective, R. N. SWANSON (éd.), Studies in Church History, 33, Woodbridge, Boydell, 1997, p. 115-134.

VAN HouTs, Elisabeth M. C., «Une hypothèse sur l'identification de Willelmus notarius comme l'historien Guillaume de Jumièges », Tabularia, http://www.unicaen.fr/ $\mathrm{mrsh} / \mathrm{crahm} /$ revue/tabularia/debatevha.html

Pour le De moribus et actis primorum Normanniae ducum de Dudon de SaintQuentin, nous restons encore tributaires de l'édition ancienne de Jules Lair ${ }^{3}$, dont une traduction anglaise a été donnée par Eric Christiansen en 1998 (une version électronique due à Felice Lifshitz, d'après le manuscrit de Berlin, est également accessible). Les nombreuses études réalisées au cours des dix dernières années ont montré combien avait été excessive la critique de cette œuvre fondamentale, faite au début

3. Dudon de SAINT-QUENTIN, De gestis Normanniae ducum seu de moribus et actis primorum Normanniae ducum, éd. Jules LAIR, Caen (Mémoires de la Société des Antiquaires de Normandie, t. XXIII/2), $1865,301 \mathrm{p}$. 
du siècle par Henri Prentout ${ }^{4}$ et relayée jusqu'à une date très récente. L'œuvre du chanoine de Saint-Quentin s'avère ainsi d'une richesse naguère insoupçonnée qui justifie de manière pressante la nécessaire réédition du texte à laquelle se consacre Pierre Bouet.

Sources éditées, publiées et/ou traduites:

Dudo of SAINT-QUENTIN, History of the Normans, trad. par Eric CHRISTIANSEN, Woodbridge, The Boydell Press, 1998, 260 p. Version électronique du manuscrit de Berlin par Felice Lifshitz: http://orb.rhodes.edu/ORB_done/Dudo/dudintro. html

Études

Albu Hanawalt, Emily, "Dudo of Saint-Quentin: The Heroic Past Imagined», Haskins Society Journal, 6, 1994, p. 111-118.

BOUET, Pierre, «Dudon de Saint-Quentin et Virgile: l'«Enéide» au service de la cause normande », in Recueil d'études en hommage à Lucien Musset (Cahier des Annales de Normandie, $n^{\circ} 23$ ), Caen, 1990, p. 215-236.

BOUET, Pierre, «Dudon de Saint-Quentin et le martyre de Guillaume Longue Épée », Les Saints dans la Normandie médiévale, Actes du colloque de Cerisy-la-Salle (26-29 septembre 1996), Pierre BouET et François NEVEuX (éd.), Caen, Presses Universitaires de Caen, 2000, p. 237-258.

CAROZZI, Claude, «Des Daces aux Normands, le mythe et l'identification d'un peuple chez Dudon de Saint-Quentin ", Peuples du Moyen Âge. Problèmes d'identification, Séminaire Société, Idéologies et Croyances au Moyen Âge [1993-1994], Claude CAROZZI et Huguette TAVIani-CarozZi (dir.), Aix-en-Provence, Publications de l'Université de Provence, 1996, p. 7-25.

Dudone di San Quintino, Pado GatTi et Antonella Degl'InNocenti (éd.), Trente, Universita degli Studi di Trento (Labirinti, 16), 1995, 218 p.

Gouttebroze, Jean-Guy, «La violence peut-elle fonder un état? Dudon de SaintQuentin et l'établissement du duché de Normandie», in La violence dans le monde médiéval (Senefiance, 36), Aix-en-Provence, CUERMA, 1994, p. 255-264.

JORDAN, Victoria B., «The Role of Kingship in Tenth Century Normandy: Hagiography of Dudo of Saint-Quentin », Haskins Society Journal, 3, 1991, p. 53-62.

LifSHITZ, Felice, «Dudo's Historical Narrative and the Norman Succession of 996 », Journal of Medieval History, 20, 1994, p. 101-120.

LIFSHITZ, Felice, «Translating "Feudal” Vocabulary: Dudo of Saint-Quentin », Haskins Society Journal, 9, 1997, Woodbridge, Boydell Press, 2001, p. 39-56.

PlassmanN, Alheydis, «Der Wandel des normannischen Geschichtsbildes im XI. Jahrhundert. Eine Quellenstudie zu Dudo von St Quentin und Wilhelm von Jumièges ", Historisches Jahrbuch, 115, 1995, p. 188-207.

4. Prentout, Henri, Étude critique sur Dudon de Saint-Quentin et son histoire des premiers ducs normands, Paris, Picard, 1916, 490 p. 
La chronique de Robert de Torigni n'a pas fait l'objet d'une sollicitude comparable, bien que l'intérêt des généalogies insérées par le chroniqueur dans les Gesta Normannorum ducum ait été réévalué ${ }^{5}$. Le Roman de Rou, dont une traduction anglaise vient d'être publiée, a été réexaminé dans une perspective soulignant l'importance et la validité des traditions orales recueillies par Wace. Au-delà, la production de ces deux auteurs, et de leur contemporain Benoît de Sainte-Maure, a été replacée dans les perspectives débattues par les travaux consacrés à la cour et à la culture politique du monde plantagenêt. Les intentions politiques qui procèdent à l'écriture - ou la réécriture - de l'histoire auraient ainsi visé à glorifier les ancêtres maternels d'Henri II, afin de rattacher les Plantagenêts à la Normandie et de justifier leurs droits sur le trône anglais, contribuant par la même à lever le vieil antagonisme entre les Normands et les Angevins et à pourvoir la dynastie d'une mémoire normande ${ }^{6}$. Inversement, la volonté de concilier une narration impartiale des faits contemporains avec un soutien effectif du roi plantagenêt a pu conduire Robert de Torigni à privilégier le genre annalistique pour la rédaction de sa chronique universelle.

Sources éditées, publiées et/ou traduites:

WACE, The Roman de Rou, trad. Glyn S. BURGESS, Saint-Hélier, Société Jersiaise, 2002, LII +408 p.

Études:

DAmian-Grint, Peter, The New Historians of the Twelfth-Century Renaissance: inventing vernacular authority, Woodbridge, Boydell and Brewer, 1999, $304 \mathrm{p}$.

Gouttebroze, Jean-Guy, « Pourquoi congédier un historiographe: Henri II Plantagenêt et Wace (1155-1174)», Romania, t. 112, 1992, p. 289-311.

KEATS-RoHAN, Katharine S. B., "Aspects of Robert of Torigny's Genealogies Revisited», Notthingham Medieval Studies, XXXVII, 1993, p. 21-27.

VAN HouTS, Elisabeth M. C., «Le roi et son historien: Henri II Plantagenêt et Robert de Torigni, abbé du Mont-Saint-Michel ", in Henri II Plantagenêt et son temps. Actes du colloque de Fontevraud, 29 septembre- $1^{\text {er }}$ octobre 1990, Cahiers de civilisation médiévale, 37, 1-2, 1994, p. 115-118.

VAn Houts, Elisabeth M. C., «Wace as Historian», in Family Trees and the Roots of Politics. The Prosopography of Britain and France from the Tenth to the Twelfth Century, Katharine Keats-Rohan (éd.), Woodbridge, The Boydell Press, 1997, p. 103-132 (rééd.

5. VAN HOUTS, Elisabeth M. C., «Robert of Torigni as Genealogist», in Studies in Medieval History presented to R. Allen Brown, Christopher HARPER-BILL, Christopher HOLDSWORTH et Janet L. NELSON (éd.) Woodbridge, The Boydell Press, 1989, p. 215-233 (rééd. in VAN HouTs, Elisabeth M. C., History and Family Traditions in England and the Continent, 1000-1200, Aldershot-Brookfield, Ashgate (Variorum Collected Studies Series), 1999, nºXII)).

6. AURELL, Martin, «La cour Plantagenêt (1154-1189): entoutrage, savoir et civilité», in La cour Plantagenêt (1154-1204). Actes du colloque tenu à Thouars du 30 avril au 2 mai 1999, MartinAurELL (dir.), Poitiers, Centre d'études supérieures de civilisation médiévale, 2000, p. 9-46. 
in VAN HOUTS, Elisabeth M. C., History and Family Traditions in England and the Continent, 1000-1200, Aldershot-Brookfield, Ashgate (Variorum Collected Studies Series), 1999, nº X).

VIELLIARD, Françoise, «Deux historiens normands du XII siècle, Guillaume de SaintPair et Wace", Revue de l'Avranchin et du Pays de Granville, t. 78, nº 389 (décembre 2001), p. 325-352.

Un autre effet positif de cette réévaluation a été la redécouverte - voire simplement la découverte - des sources en marge de la «grande» historiogaphie normande et des foyers qui les ont abritées. Jacques Le Maho a ainsi attiré l'attention sur une curieuse «notice» rapportant la venue de personnages originaires d'Asiniaca (probablement Isigny-sur-Mer ${ }^{7}$ ) sur le site de l'ancien monastère détruit de Fécamp ${ }^{8}$. Le texte, attribuable à un homme d'Église (peut-être Henri, évêque de Bayeux) au fait des traditions fécampoises, serait dans l'état actuel de la recherche la plus ancienne pièce écrite composée dans le duché, au début des années 930. L'élaboration de cette première historiographie fécampoise est ponctuée de jalons qui commencent maintenant à être mieux connus, telle la chronique dite du «manuscrit Rouen 528» rapportant la fondation de l'abbaye et probablement composée peu avant 1001. L'abbaye semble aussi avoir rassemblé des matériaux parfois d'origines très diverses, dont se servirent ultérieurement les chroniqueurs anglo-normands. Elisabeth Van Houts a ainsi exhumé un document capital pour comprendre l'organisation logistique de la conquête de l'Angleterre, en l'occurrence la liste des principaux contributeurs à l'expédition avec l'indication du nombre de vaisseaux fournis ${ }^{9}$. Le texte, probablement rédigé à Fécamp sur la base d'informations recueillies dans les mois précédant l'appareillage de la flotte normande, suggère non seulement le rôle joué par Fécamp dans l'organisation des opérations mais aussi dans la conservation et la transmission de la mémoire écrite ou orale des événements. Dans les années 1160/1170, Wace puisa à Fécamp une partie de son information sur la conquête tandis que la liste des navires fournis par les grands de Normandie était copiée, au plus tard vers 1160 dans un manuscrit de l'abbaye de Battle. Cette dernière, fondée par Guillaume le Conquérant sur le lieu de sa victoire sur Harold avait d'ailleurs probablement entrepris de rassembler des traditions historiographiques venues du continent et dont on retrouve la trace dans la Brevis relatio de Guillelmo nobilissimo comite Normannorum.

7. Calvados, ch.-1. de cant.

8. LE MAHO, Jacques, «Un exode de reliques dans les pays de la Basse Seine à la fin du IX esiècle», Bulletin de la Commission départementale des Antiquités de Seine-Maritime, t. XLVI, 1998, p.174-181; $i d$. «Les origines de l'abbaye de Fécamp, de l'histoire à la légende», De l'histoire à la légende. La broderie du Précieux-Sang, Catalogue de l'exposition tenue à Fécamp, avril-juin 2001, Fécamp, 2001, p. $25-26$.

9. VAN HOUTS, Elisabeth M. C., «The ship list of William the Conqueror », Anglo-Norman Studies, X, 1987, p. 159-183 (rééd. in VAN HOUTS, Elisabeth M. C., History and Family Traditions in England and the Continent, 1000-1200, Aldershot-Brookfield, Ashgate (Variorum Collected Studies Series), $\left.1999, \mathrm{n}^{\circ} \mathrm{VI}\right)$ 
Sources éditées, publiées et/ou traduites:

The Brevis relatio de Guillelmo nobilissimo comite Normannorum, éd. Elisabeth M. C. VAN HOUTS, Camden fifth series, vol. 10, «Chronology, Conquest and Conflict in Medieval England: Camden Miscellany XXXIV», 1997, p. 1-48 (rééd. in VAN HOUTS, Elisabeth M. C., History and Family Traditions in England and the Continent, 1000-1200, Aldershot-Brookfield, Ashgate (Variorum Collected Studies Series), 1999, n VII).

Chronique dite du Manuscrit Rouen 128, éd. Mathieu ARNOUX, "Before the Gesta Normannorum and beyond Dudo: some evidence on early Norman historiography", Anglo-Norman Studies, XXII, 1999, p. 19-48 (éd. du texte p. 43-46).

\section{Études}

ARNOUX, Mathieu, «La fortune du Libellus de revelatione, edificatione et auctoritate Fiscannensis monasterii. Note sur la production historiographique d'une abbaye bénédictine normande", Revue d'Histoire des Textes, t. XXI, 1991, p. 135-158.

ARNOUX, Mathieu, «Before the Gesta Normannorum and beyond Dudo: some evidence on early Norman historiography», Anglo-Norman Studies, XXII, 1999, p. 29-48.

ARnoux, Mathieu, «Les premières chroniques de Fécamp: de l'hagiographie à l'histoire ", Les Saints dans la Normandie médiévale, Actes du colloque de Cerisy-laSalle (26-29 septembre 1996), Pierre BouET et François Neveux (éd.), Caen, Presses Universitaires de Caen, 2000, p. 71-82.

D'autres foyers, comme Rouen ou le Mont-Saint-Michel ${ }^{10}$, ont bénéficié d'une sollicitude particulière que viendront sans doute renforcer la parution des travaux consacrés aux sources hagiographiques normandes dans les actes du colloque de Cerisy-la-Salle sur les saints dans la Normandie médiévale, les dossiers consacrés à la production de Saint-Wandrille et de Jumièges dans le volume des Beihefte der Francia sur l'hagiographie du haut Moyen Âge en Gaule du Nord et la récente réédition des Gesta des abbés de Fontenelle ${ }^{11}$. Trois pièces produites dans le milieu normanno-poitevin qui contribua au relèvement de l'abbaye de Jumièges, la Vie de saint Aycadre, celle de saint Hugues et la Complainte de Guillaume Longue Épée, font également l'objet de recherches qui apportent un regard neuf sur la régence de Louis IV en Normandie et viennent souligner le rôle tenu par Jumièges dans le renouveau de la production narrative au milieu du $\mathrm{X}^{\mathrm{e}}$ siècle. C'est également à

10. Avec en particulier la préparation, par Pierre Bouet et Olivier Desbordes, de l'édition de la Revelatio, de l'Introductio monachorum et du De scuto et gladio de Baudry de Bourgueil ainsi que celle du Roman $d u$ Mont-Saint-Michel de Guillaume de Saint-Pair par Catherine Bougy; BouET, Pierre, «La Reuelatio ecclesiae sancti Michaelis et les origines du culte de saint Michel sur le Mont Tombe », in Cultes et pèlerinage à saint Michel en Occident, Actes du colloque de Cerisy-la-Salle (2000), à paraître.

11. Pour tous ces travaux, nous renvoyons à la bibliographie placée à la fin de cet article. Sur le foyer historiographique de Saint-Wandrille, voir également: VAN HOUTS, Elisabeth M. C., «Historiography and Hagiography at Saint-Wandrille: the "Inventio et Miracula Sancti Vulfranni" », Anglo-Norman Studies, XII, 1989, p. 233-251 (rééd. in VAN HouTs, Elisabeth M. C., History and Family Traditions in England and the Continent, 1000-1200, Aldershot-Brookfield, Ashgate (Variorum Collected Studies Series), 1999, $\mathrm{n}^{\circ}$ IV). 
cette période, postérieure à l'assassinat de Guillaume Longue Épée, que Felice Lifshitz rattache la Vita Romani composée par un certain Fulbert, dont l'identité et la chronologie ne recueillent pas l'unanimité des spécialistes. Si les résultats de cette enquête sur le dossier de la vie de saint Romain sont contestés, ils ont toutefois le mérite de poser de bonnes questions sur la genèse de l'identité normande et le rôle joué par les archevêques de Rouen dans la restauration de la culture littéraire dans le duché de Normandie.

L'examen de ces matériaux pose tout un ensemble de problèmes qui nuancent considérablement des idées bien établies, particulièrement sur le premier siècle de l'histoire du duché. Il invite d'une part à réviser l'impression de « désert culturel» traditionnellement accolé à la principauté normande jusqu'aux alentours de l'an mil. D'autre part, l'historien est conduit à s'interroger sur le besoin d'histoire qui semble bien caractériser les élites civiles et ecclésiastiques du duché. Il est maintenant évident que la première œuvre historiographique majeure composée en Normandie, par Dudon de Saint-Quentin, n'a pas été une entreprise isolée et que l'intense effort de fixation de la mémoire par l'écrit, visible dans la dernière décennie du $\mathrm{X}^{\mathrm{e}}$ siècle, correspond à une période de profonds changements dans la société et l'État normands. De ce point de vue, le champ des interprétations reste ouvert, mais nous sommes tentés d'y voir là l'expression d'une assimilation réussie des Normands dans la civilisation de l'Europe chrétienne et les anxiétés engendrées par cette situation; de montrer aux descendants des Scandinaves établis en Neustrie, désormais devenus des Francs, qu'ils sont bien des Normands, détenteurs d'une identité qui les place à part dans le concert des nations de l'Occident.

Sources éditées, publiées et/ou traduites:

ARNOUX, Mathieu, «La conversion des Normands de Neustrie et la restauration de l'Église dans la province de Rouen", in Le christianisme en Occident du début du $V I I^{e}$ siècle au milieu du XIe siècle. Textes et documents réunis par François BOUGARD, Paris, SEDES, 1997, p. 269-281.

Carmen de fundatione, ruina et restauratione inclyti monasterii Gemmeticensis, éd. et trad. Pierre BOUET, Tabularia «Études», $n^{\circ}$ 1, 2001, p. 33-52.

Chronique des abbés de Fontenelle (Saint-Wandrille), éd. Pascal Pradié, Paris, Les Belles-Lettres ( «Les classiques de l'Histoire de France au Moyen Âge»), 1999, 283 p.

Édition électronique de: The Planctus for William Longsword, Robert HeLMERICHS (éd), http://www.ukans.edu/carrie/Planctus).

Études :

BAUDUIN, Pierre, «Autour d'une construction identitaire: la naissance d'une historiographie normande à la charnière des $\mathrm{X}^{\mathrm{e}}-\mathrm{XI}^{\mathrm{e}}$ siècles", in Conquête, acculturation, identité: des Normands aux Hongrois. Les traces de la conquête, textes rassemblés et présentés par Piroska NAGY, Cahiers du GRHIS, 13 (2001), p. 79-91.

BOUET, Pierre, «Les sources hagiographiques: nature et méthode d'analyse», Les Saints dans la Normandie médiévale, Actes du colloque de Cerisy-la-Salle (26-29 septembre 
1996), Pierre Bouet et François Neveux (éd.), Caen, Presses Universitaires de Caen, 2000, p. 11-20.

BOUET, Pierre, «Bibliographie des sources relatives aux saints normands », Les Saints dans la Normandie médiévale, Actes du colloque de Cerisy-la-Salle (26-29 septembre 1996), Pierre Bouet et François NeveuX (éd.), Caen, Presses Universitaires de Caen, 2000, p. 305-323.

BOUET, Pierre, «Les chroniqueurs francs et normands face aux invasions vikings », in L'Héritage maritime des Vikings en Europe de l'Ouest, Actes du colloque international de la Hague (Flottemanville-Hague, 30 septembre-3 octobre 1999), Elisabeth RidEL (éd.), Caen, Presses Universitaires de Caen, 2002, p. 57-74.

BougY, Catherine, "Comment les chroniqueurs du XII siècle ont-ils perçu les invasions vikings? ", in L'Héritage maritime des Vikings en Europe de l'Ouest, Actes du colloque international de la Hague (Flottemanville-Hague, 30 septembre-3octobre 1999), Elisabeth RIDEL (éd.), Caen, Presses Universitaires de Caen, 2002, p. 75-100.

GAUTHIER, Nancy, «Quelques hypothèses sur la rédaction des vies des saints évêques de Normandie ", Memoriam sanctorum venerantes. Miscellanea in onore di Monsignor Victor Saxer, Vatican, Pontifico Istituto di archeologia cristiana, Cité du Vatican, 1992, p. $449-468$.

HowE, John, «The Hagiography of Jumièges (Province of Haute-Normandie) », in L'hagiographie du haut Moyen Âge en Gaule du Nord. Manuscrits, textes et centres de production, Martin HeinzelmanN (dir.), Stuttgart, Thorbecke (Beihefte der Francia, 52), 2001, p. 91-125.

HowE, John, «The hagiography of Saint-Wandrille (Fontenelle)», in L'hagiographie du haut Moyen Âge en Gaule du Nord. Manuscrits, textes et centres de production, Martin HeinZelmanN (dir.), Stuttgart, Thorbecke (Beihefte der Francia, 52), 2001, p. 127-192.

LE MAHO, Jacques, «La production éditoriale à Jumièges vers le milieu du $\mathrm{X}^{e}$ siècle », Tabularia «Études», $\mathrm{n}^{\circ}$ 1, 2001, p. 11-32.

LIFSHITZ, Felice, «The Acta archiepiscoporum Rotomagensium: a Monastery or Cathedral Product? », Analecta Bollandiana, t. 108, 1990, p. 337-347.

LIFSHITZ, Felice, «St Romain de Rouen : missionnaire franc dans la Normandie des Vikings ", Voix d'ouest en Europe. Souffles d'Europe en ouest. Actes du colloque international d'Angers, 21-24 mai 1992, Presses de l'Université d'Angers, 1993, p. 23-30.

LifSHITZ, Felice, The Norman Conquest of Pious Neustria. Historiographic Discourse and Saintly Relics 684-1090, Toronto, Pontifical Institute of Mediaeval Studies, 1995, $324 \mathrm{p}$.

POTTS, Cassandra, «Atque unum ex diversis gentibus populum effecit: historical tradition and the norman identity", Anglo-Norman Studies, XVIII, 1995, p. 139-152.

VAn HouTs, Elisabeth M. C., "A Note on Jezabel and Semiramis, Two Latin Norman Poems from the Early Eleventh Century, Journal of Medieval Latin, 2, 1992, p. 18-24 (rééd. in VAN HouTs, Elisabeth M. C., History and Family Traditions in England and the Continent, 1000-1200, Aldershot-Brookfield, Ashgate (Variorum Collected Studies Series), 1999, n IIIa).

VAN HoUTS, Elisabeth M. C., "The Date of Warner of Rouen's Moriuth», in VAN HouTs, Elisabeth M. C., History and Family Traditions in England and the Continent, 1000-1200, Aldershot-Brookfield, Ashgate (Variorum Collected Studies Series), 1999, $\left.\mathrm{n}^{\circ} \mathrm{IIIb}\right)$. 


\begin{abstract}
VAughn, Sally N., "Among These Authors are the Men of Bec: Historical Writing among the Monks of Bec », Essays in Medieval Studies, 17, 2000, 13 p.

http://www.luc.edu/publications/medieval/vol17/vaughn.html

VIOLETTE, Louis, «Le problème de l'attribution d'un texte rouennais du XIe siècle: les Acta archiepiscoporum Rothomagensium », Analecta Bollandiana, 115, 1997, p. 113129.

VIOLETTE, Louis, «Le thème des origines de l'Église de Rouen d'après une œuvre de ses chanoines: les Annales de Rouen", in Chapitres et cathédrales en Normandie, Actes du XXXI ${ }^{e}$ Congrès des Sociétés historiques et archéologiques de Normandie, Sylvette Lemagnen et Philippe Manneville (éd.), Annales de Normandie, Série des Congrès des Sociétés historiques et archéologiques de Normandie, Caen, II, 1997, p. 287294.

ViOLETTE, Louis, «Une entreprise historiographique au temps de la Réforme grégorienne: les Actes des archevêques de Rouen", Revue d'Histoire de l'Église de France, t. 83, juil.-déc. 1997, p. 343-365.
\end{abstract}

\title{
2. Les sources diplomatiques et la «mutation documentaire » du $\mathrm{XI}^{\mathrm{e}}$ siècle
}

Écrire l'histoire n'est pas simplement la préoccupation des chroniqueurs. David Bates a souligné que plusieurs des pancartes et grandes chartes de confirmation normandes pouvaient ressembler à une sorte d'œuvre littéraire. Les deux versions de la grande charte de dotation de la collégiale d'Auchy, rédigées à la fin du XIe siècle, retracent chacune à leur manière l'établissement de la domination normande sur les confins picards de la Normandie ${ }^{12}$. Les recherches conduites sur les cartulaires du Mont-Saint-Michel, de Notre-Dame et Mortemer, de Saint-Pierre de Préaux et de Saint-Martin de Troarn montrent que la rédaction d'un cartulaire ne procède pas uniquement d'un souci de gestion mais aussi d'une tentation d'écrire l'histoire des établissements concernés. La familiarité des praticiens de l'écrit documentaire avec la production historiographique n'est plus à démontrer. Ce sont souvent les mêmes hommes qui œuvrent. L'auteur anonyme des Gesta des abbés de Fontenelle était probablement le moine chargé de la garde des archives, dans lesquelles il puisa abondamment pour alimenter son œuvre. On sait que Dudon de Saint-Quentin produisit des diplômes ducaux; dans l'un des deux actes originaux portant sa souscription, l'auteur du De moribus et actis primorum Nomanniae ducum se qualifie lui-même de cancellarius.

Il est habituel, lorsque l'on aborde la production documentaire, de rappeler les propos tenus par Léopold Delisle appelant de ses vœux, en conclusion de son discours prononcé devant la Société des Antiquaires de Normandie le 4 décembre 1862,

12. Rouen, Arch. dép. Seine-Maritime, 1 H 1, 1 H 13. Sur ce point, BAUduIN, Pierre, La Première Normandie ( $X^{e}-X I^{e}$ siècles). Sur les frontières de la haute Normandie: identité et construction d'une principauté, Caen, Presses Universitaires de Caen (sous presse). 
la nécessaire publication des sources normandes ${ }^{13}$. Les échos de cette demande se rencontrent encore dans des articles publiés ces dernières années. Cassandra Potts résumait bien la situation en 1992 : «En dehors du corpus des chartes ducales, le nombre des chartes privées se rapportant à la Normandie du XI ${ }^{\mathrm{e}}$ siècle est impressionnant. Bien qu'un grand nombre ait été publié, des centaines restent inédites en France, dans les archives, les bibliothèques et les collections privées ». A l'occasion de la table ronde sur les actes comme expression du pouvoir au haut Moyen Âge, en novembre 1999, David Bates soulignait les difficultés engendrées par l'analyse des documents normands du $\mathrm{XI}^{\mathrm{e}}$ siècle en raison d'un nombre encore élevé d'actes inédits, dont une grande proportion de notices.

La publication par David Bates, en 1998, des actes de Guillaume le Conquérant postérieurs à la conquête de l'Angleterre est venue compléter les recueils de Marie Fauroux ${ }^{14}$ et de Lucien Musset ${ }^{15}$, soit au total près de 340 actes ducaux couvrant la quasi-totalité des $\mathrm{X}^{\mathrm{e}}$-XI ${ }^{\mathrm{e}}$ siècles (jusqu'en 1087). Au-delà de cette date, nous sommes encore redevables de publications anciennes (dues à Charles Homer Haskins ${ }^{16}$, à Léopold Delisle et Elie Berger ${ }^{17}$ ) ou de thèses demeurées inédites comme celles d'Henry Chanteux ${ }^{18}$ et de Claude Fagnen ${ }^{19}$, respectivement sur les actes normands d'Henri Beauclerc et de Richard Cœur de Lion. Des projets de publication sont toutefois en cours, concernant les actes de Robert Courteheuse (par une équipe associant des chercheurs français (P. Bauduin, V. Gazeau) et britanniques (D. Bates, J. Green, E. Van Houts)), d'Henri II Plantagenêt et de Richard Cœur de Lion ${ }^{20}$ (par Nicholas Vincent), tandis que des études ont porté plus particulièrement sur les chartes de Mathilde l'Emperesse et dernièrement d'Henri le Jeune.

13. DelisLe, Léopold, «Discours d'ouverture de la séance publique du 4 décembre 1862 », Bulletin de la Société des Antiquaires de Normandie, II, 1862, p. 304-337, à la p. 336-337: «En publiant nos anciennes chartes, vous resterez fidèles aux inspirations qui ont présidé à la fondation de la Société ; vous arracherez à l'oubli les restes d'un passé glorieux; vous établirez sur des bases inébranlables l'histoire de la Normandie; vous mettrez dans tout son jour la part que nos pères ont prise à développer la civilisation du moyen-âge et à préparer les progrès des temps modernes ». Cette citation est reprise par David Bates dans l'introduction de son édition des actes de Guillaume le Conquérant.

14. Cité note 1 .

15. Musset, Lucien, Les actes de Guillaume le Conquérant et de la reine Mathilde pour les abbayes caennaises, Caen (Mémoires de la Société des Antiquaires de Normandie, t. XXXVII), 1967, 180 p.

16. HASKINS, Charles Homer, Norman Institutions, Cambridge Mass., Harvard University Press, 1918, $377 \mathrm{p}$.

17. DelisLe Léopold, Berger, Elie, Recueil des actes d'Henri II, roi d'Angleterre et duc de Normandie concernant les provinces françaises et les affaires de France, 4 vol., Paris, Académie des Inscriptions et BellesLettres, 1909-1924.

18. ChanteuX, Henry, Recueil des actes d'Henri Ier Beauclerc, duc de Normandie, Thèse de l'École nationale des chartes, Paris, 1932, 3 vol. (dactyl.).

19. FAGNEN, Claude, Essai sur quelques actes normands de Richard Cour-de-Lion, Thèse de l'École nationale des chartes, Paris, 1971, 5 vol. (dactyl.).

20. Mortimer, Richard, "The charters of Henry II : what are the criteria for authenticity? ", AngloNorman Studies, XII, 1989, p. 119-134. «The Angevin Acta Project in France and England », AngloNorman Anonymous, 13, 3, octobre 1995. 
Sources éditées, publiées et/ou traduites:

BATES, David, Regesta Regum Anglo-Normannorum. The Acta of William I (1066-1087), Oxford, Clarendon Press, 1998, XXXVIII + 1153 p.

Études :

BATES, David, «The Conqueror's Charters», in England in the Eleventh Century, Carola Hicks (éd.), Stamford, Paul Watkins (Harlaxton Medieval Studies, vol. II), 1992, p. 1-15.

ChIBNALl, Marjorie, "The charters of the Empress Mathilda ", Law and Government in Medieval England, Essays in honour of Sir James Holt, Georges GARNETT et John HUdSON (éd.), Cambridge, Cambridge University Press, 1994, p. 276-298.

SMITH, R. J., «Henry II's heir: the Acta and Seal of Henry the Young King, 1170-1183», The English Historical Review, CXVI, ${ }^{\circ}$ 466, avril 2001, p. 297-326.

La publication des autres pièces de la production diplomatique normande n'est pas aussi avancée. Des outils de travail existent, à commencer par les fascicules consacrés aux sources de l'histoire de la Normandie à la Bibliothèque nationale publiés par Michel Nortier ${ }^{21}$. Le «catalogue des sources de l'histoire domaniale » dressé par Lucien Musset en $1945^{22}$ englobe le $\mathrm{X}^{\mathrm{e}}$ siècle normand, c'est-à-dire très peu de chose (une dizaine de pièces) compte tenu du vide documentaire qui caractérise les premières décennies de l'histoire du duché. La liste des actes du XI ${ }^{\mathrm{e}}$ siècle établie par Emily-Zack Tabuteau en $1988^{23}$, sans prétendre à l'exhaustivité, recense quelque 888 actes, dont $40 \%$ étaient alors demeurés inédits et un grand nombre publiés anciennement. Cette estimation de l'ordre de 30 à $40 \%$ de pièces inédites, pour le seul XIe siècle, donne une idée de la tâche qui reste à accomplir. Diverses initiatives ont d'ores et déjà permis de combler certains retards les plus criants. Ces dernières années ont vu la publication d'une documentation importante sur l'abbaye de la Trinité de Caen, les établissements de chanoines réguliers de la province de Rouen, les abbayes de Notre-Dame-du-Val et du Val-Richer. Il faut y ajouter les parutions imminentes ou annoncées des cartulaires de l'abbaye Saint-Pierre de Préaux, du Mont-Saint-Michel, du chartrier de la cathédrale de Coutances, des chartes de l'abbaye de Conches (par Claire de Haas) et celles toujours espérées du cartulaire de Saint-Martin de Sées ${ }^{24}$ ou du chartier de Savigny ${ }^{25}$. Les correspondances des dignitaires

21. Nous renvoyons à la «Bibliographie des travaux de Michel Nortier», in Recueil d'études normandes offert en hommage à Michel Nortier, Cahiers Léopold Delisle, t. XLIV, 1995, p. 5-27.

22. MusSET, Lucien, «Notes pour servir d'introduction à l'histoire foncière de la Normandie. Les domaines de l'époque franque et les destinées du régime domanial du $\mathrm{IX}^{\mathrm{e}}$ au XI $\mathrm{XI}^{\mathrm{e}}$ siècle », Bulletin de la Société des Antiquaires de Normandie, XLIX, 1942-1945, p. 7-97.

23. TABUteau, Emily Zack, Transfers of Property in Eleventh-Century Norman Law, Chapell-Hill et Londres, University of North California Press, 1988, p. 250-268.

24. BOUVRIS, Jean-Michel, «Aux premiers temps d'une grande abbaye normande au $\mathrm{XI}^{\mathrm{e}}$ siècle: les chartes de fondation de Saint-Martin de Sées ", Annales de Normandie, 39e année, 4, 1989, p. 452-454.

25. POulle, Béatrice, Le chartrier de l'abbaye de Savigny au diocèse d'Avranches : édition partielle (1202 1243) et commentaires, Thèse de l’École nationale des chartes, Paris, 1989, 537 p. (dactyl). 
ecclésiastiques normands ou anglo-normands (Anselme, Thomas Becket, Arnoul de Lisieux) ont également fait l'objet d'une sollicitude grandissante.

Sources éditées, publiées et/ou traduites:

Des clercs au service de la réforme. Études et documents sur les chanoines réguliers de la province de Rouen, Mathieu ARNOUX (dir.), Turnhout, Brepols (Bibliotheca Victorina XI), 2000 (appendice documentaire, p. 273-367, qui contient également les Chronique de Sainte-Barbe-en-Auge, p. 275-293, Chronique de fondation de l'Ile-Dieu, p. 297-306, Chronique de fondation de Notre-Dame d'Ardenne, p. 306-310).

ARNOUX, Mathieu, "Cartulaire de la sidérurgie normande (1085-1337)", in Mineurs, férons et maîtres de forge. Études sur la production du fer dans la Normandie du Moyen Âge, $X I^{e}-X V^{e}$ s., Paris, C.T.H.S., 1993, p. 419-484.

ARNOUX, Mathieu, «Aux origines d'une léproserie : la pancarte de la Madeleine d'Orbec (1105-1135)", in Recueil d'études normandes offert en hommage à Michel Nortier, Cahiers Léopold Delisle, t. XLIV, 1995, p. 209-222.

ARnoux, Mathieu, MANEUVRIER, Christophe, «Deux abbayes de Basse-Normandie: Notre-Dame du Val et le Val Richer (XII ${ }^{\mathrm{e}}$-XIII ${ }^{\mathrm{e}}$ siècles) », Flers, Le Pays Bas Normand, $\mathrm{n}^{\circ} 237-238,2000(1-2), 111 \mathrm{p}$.

BATES, David, «Four recently rediscovered charters », Annales de Normandie, 45, $\mathrm{n}^{\circ} 1$, mars 1995, p. 35-48.

DoR, Marc-Antoine, Seigneurs en Ile-de-France occidentale et en Haute-Normandie: contribution à l'histoire des seigneurs de Montfort-l'Amaury, des comtes d'Evreux, et de leur entourage au XII e siècle et au début du XIII siècle, Thèse de l'École nationale des chartes, Paris, 1992, 2 vol. (dactyl.).

EVERARD, Judith et JONES, Michael, The Charters of Duchess Constance of Brittany and her Family, 1171-1221, Woodbridge, The Boydell Press, 2000, 217 p.

FONTANEL, Julie, Le chartrier de la cathédrale de Coutances, XI ${ }^{e}$-XIVe siècles. Étude et édition. Thèse pour le diplôme d'archiviste paléographe, Paris, École nationale des chartes, 1999, 4 vol. 906 p. (dactyl.).

Keats-Rohan, Katharine S. B., The Cartulary of Mont-Saint-Michel, Stamford, Paul Watkins Publishing, (à paraître).

RouET, Dominique, Le cartulaire de l'abbaye Saint-Pierre de Préaux: étude et édition du manuscrit dans son état de 1227, Thèse pour le diplôme d'archiviste paléographe, Paris, École nationale des chartes, 1999, 4 vol., 896 p. (dactyl.) (à paraître aux éditions du CTHS).

WALMSLEY, John, Charters and custumals of the abbey of Holy Trinity Caen. Part 2 The French Estates, Oxford University Press, 1994 (Records of social and economic history, new series 22), $160 \mathrm{p}$.

MÜLLER, Harald, Päpstlische Delegationsgerichtsbarkeit in der Normandie, 12. und frühes 13. Jahrhundert, Bonn, Bouvier, 1997, 2 vol., X + 285 p., 503 p.

dont correspondances : 
The Letters of Saint Anselm of Canterbury, trad. et notes de Walter FRÖHLICH, Cistercian Publications (96, 97, 142), Kalamazoo (Michigan), 1990-1994, 3 vol., 354, 347 et $304 \mathrm{p}$.

The letter collections of Arnulf of Lisieux, trad. Carolyn Poling SCHRIBER, Lewiston (N.Y.), Mellen Press, 1997, XII + 337 p.

The Correspondence of Thomas Becket, Archbishop of Canterbury, 1162-1170, éd. et trad. Anne J. DugGan, Oxford, Clarendon Press (Oxford Medieval Texts), 2000, 2 vol., CLXXXII + 1490 p.

Études :

FONTANEL, Julie, «Le chartrier de la cathédrale de Coutances, $\mathrm{XI}^{\mathrm{e}}-\mathrm{XIV}^{\mathrm{e}}$ siècle», Position des thèses..., Paris, École nationale des chartes, 2000, p. 143-148.

KeATS-ROHAN, Katharine, «Bibliothèque municipale d'Avranches, 210: Cartulary of Mont-Saint-Michel », Anglo-Norman Studies, XXI, 1998, p. 95-112.

LOUISE, Gérard, «Recherches sur le cartulaire perdu de l'abbaye bénédictine de Lonlay (Basse-Normandie, Orne) », Le Domfrontais médiéval, 15, A.R.C.D., Domfront, 2000, p. 4-13.

Poulle, Béatrice, «Les sources de l'histoire de l'abbaye cistercienne de Savigny (Manche) au diocèse d'Avranches », Revue Mabillon, nouvelle série, 7 (t. 68), 1996, p. 105125 .

Rouet, Dominique, «Le cartulaire de Saint-Pierre de Préaux. Étude et édition du manuscrit dans son état de 1227 ", Position des thèses..., Paris, École nationale des chartes, 1999, p. 341-347.

ROUET, Dominique, «Le cartulaire de l'abbaye Saint-Pierre de Préaux: présentation du manuscrit», Tabularia «Documents», n 1, p. 1-11.

Outre la mise à la disposition des chercheurs d'un large éventail de documents, l'une des avancées les plus significatives de la recherche a été d'inscrire la production diplomatique normande dans le thème très débattu de la «mutation documentaire», un concept qui rend compte du réaménagement des formes et de la diversité des pratiques de l'écrit ainsi que de leur interprétation à l'échelle des structures politiques, sociales et culturelles. Voici une dizaine d'année, Cassandra Potts dégageait les grandes caractéristiques de l'évolution des chartes normandes antérieures à 1066, associant les transformations observables après le règne de Richard II à la rupture du contrôle public, mais aussi à un usage plus répandu de l'écrit et à une participation accrue des laïcs à la vie ecclésiastique du duché. Ses conclusions ont été depuis précisées et nuancées par l'examen de types diplomatiques particuliers tels que les pancartes, les notices, les chirographes et les conventiones. Il est maintenant acquis que, si la diversification de la documentation normande est incontestable, en particulier dans la seconde moitié du XIe siècle, il n'y a pas eu de rupture fondamentale: le développement de nouvelles formes diplomatiques ne s'est pas accompagné de la disparition des anciennes. Quant aux rapports (supposés) entre la mutation documentaire et les mutations sociales et politiques, David Bates a récemment souligné les faux semblants qui conduiraient à percevoir les chartes comme le fidèle reflet de 
l'histoire du pouvoir : l'analyse doit prendre en considération les formes complexes d'un pouvoir dont les manifestations ne sont pas forcément explicites et le développement de pratiques plus lettrées. Sans doute les recherches à venir permettront d'affiner les résultats d'une enquête dont tous les matériaux n'ont pas été examinés. Ainsi, en dépit de travaux demeurés inédits ${ }^{26}$, il n'existe par exemple aucune vue d'ensemble sur les actes épiscopaux normands. Inversement d'autres aspects de la production diplomatique normande, comme la datation des actes normands ou la diffusion, aux XII ${ }^{-}$-XIII ${ }^{e}$ siècles, des actes passés coram parrochia, ont été abordés.

Ces avancées invitent à l'exploitation des outils existants et à l'élaboration de nouveaux instruments. L'utilisation de la base de données élaborée par l'équipe «textes diplomatiques» de l'ARTEM (Atelier de recherche sur les textes médiévaux - UMR 7002 Moyen Âge/Nancy II) est familière pour ceux qui travaillent sur les actes normands des $\mathrm{XI}^{\mathrm{e}}$-début XII ${ }^{\mathrm{e}}$ siècles. L'inventaire des 5000 actes originaux antérieurs à 1121 conservés dans les dépôts français ${ }^{27}$ permet de recenser plus de 180 actes en faveur d'établissements ou de particuliers du duché ainsi que de dépendances normandes de maisons extérieures à la Normandie; auxquels il faut ajouter une douzaine de diplômes ducaux ou d'actes épiscopaux pour des bénéficiaires situés en dehors des frontières de la principauté normande. Une demi douzaine d'églises normandes présentent des séries suffisamment conséquentes (Jumièges : 34 actes, Saint-Ouen de Rouen: 33, Fécamp: 22, plus d'une douzaine d'actes: Saint-Etienne de Caen, Saint-Wandrille, Saint-Martin de Troarn) pour solliciter une étude diplomatique approfondie, comme celle menée tout récemment par Benoît-Michel Tock pour les chartes de Jumièges. Le projet «Données textuelles et production documentaire dans la Normandie médiévale » lancé en 2000 au Centre de Recherches Archéologiques et Historiques Médiévales de Caen, propose l'élaboration d'un outil de travail orientant la recherche sur les processus de production documentaire dans la Normandie médiévale. Le champ d'expérimentation retenu concerne les $\mathrm{X}^{\mathrm{e}}$-XI ${ }^{\mathrm{e}}$ siècles, ce cadre chronologique devant être à terme élargi. L'objectif principal est la réalisation d'un corpus des actes normands des $\mathrm{X}^{\mathrm{e}}$-XI ${ }^{\mathrm{e}}$ siècles. Toutefois, l'élaboration de cet instrument doit s'intégrer dans une recherche sur les conditions de la production documentaire et l'appropriation de l'écrit par une société. La démarche doit aussi prendre en compte les sources narratives, afin d'éclairer les processus de transmission de la mémoire et leurs enjeux, et s'interroger sur la manière dont l'écrit révèle les mutations sociales. La revue Tabularia, issue de ce projet, participe à cette réflexion.

26. DupuY, Hubert, Recueil des actes des évêques de Bayeux antérieurs à 1205, Thèse de l'École nationale des chartes, Paris, 1970, 2 vol. (dactyl.); WALDMAN, Thomas G., Hugh of Amiens, archbishop of Rouen (1130-1164), PhD Thesis, Université d'Oxford, 1970, 658 p. (dactyl.), avec les actes d'Hugues d'Amiens (p. 166-561).

27. La diplomatique française du Haut Moyen Âge. Inventaire des chartes originales antérieures à 1121 conservées en France, Benoît-Michel TOCK (dir), 2 vol., Turnhout, Brepols (Collection ARTEM-4), 2001, 425 et 428 p. 
ARNOUX, Mathieu, «Essor et déclin d'un type diplomatique: les actes passés coram parrochia en Normandie (XII - -XIII ${ }^{e}$ siècles) ", Bibliothèque de l'École des Chartes, 154, 2 (juillet-décembre 1996), p. 323-357.

ARNoux, Mathieu, «Disparition ou conservation des sources et abandon de l'acte écrit: quelques observations sur les actes de Jumièges », Tabularia «Études », n 1 , 2001, p. 1-10.

BATES, David, GAZEAU, Véronique, «L'abbaye de Grestain et la famille d'Herluin de Conteville», Annales de Normandie, 40e année, n 1, mars 1990, p. 5-30.

BATES, David, «Le rôle des évêques dans l'élaboration des actes ducaux et royaux entre 1066 et 1077 ", Les évêques normands du XI siècle, Actes du colloque de Cerisy-laSalle (30 sept.-3 oct. 1993), Pierre Bouet et François Neveux (dir.), Caen, Presses Universitaires de Caen, 1995, p. 103-115.

BATES, David, «The Prosopographical Study of Anglo-Norman Royal Charters », in Katharine Keats Rohan (éd.), Family Trees and the Roots of Politics : the Prosopography of Britain and France from the Tenth to the Twelfth Centuries, Woodbridge, Boydell Press, 1997, p. 89-102.

BATES, David, «Les chartes de confirmation et les pancartes normandes du règne de Guillaume le Conquérant ", Pancartes monastiques des XI ${ }^{e}$-XII ${ }^{e}$ siècles, études réunies par Michel PARISSE, Pierre PÉGEOT, Benoît-Michel Tock, Turnhout, Brepols, 1998, p. 97-109.

BATES, David, « La mutation documentaire et le royaume anglo-normand (seconde moitié du XI ${ }^{e}$ siècle-début XII ${ }^{e}$ siècle) », Les actes comme expression du pouvoir au Haut Moyen Âge, Actes de la table ronde organisée par l'ARTEM (Nancy 2), 27-29 novembre 1999, sous presse.

BOUVRIS, Jean-Michel, «A propos des échanges entre les "scriptoria” des abbayes caennaises au XIe siècle : la "donation" de la dîme de Biéville par Renouf, vicomte de Bayeux ", Recueil d'études en hommage à Lucien Musset, Cahier des Annales de Normandie, $\mathrm{n}^{\circ}$ 23, Caen, 1990, p. 203-214.

BouvrIs, Jean-Michel, «Une possession des comtes d'Eu dans le Houlme à l'époque ducale: Bazoches. A propos d'une charte originale de Jean, comte d'Eu, de 1154 », Annuaire des cinq départements de la Normandie, 153, 1995, p. 79-89.

CROUCH, David, «A Norman «conventio » and bonds of lordship in the Middle Ages», Law and Government in Medieval England, Essays in honour of Sir James Holt, Georges GARNETT et John HudSON (éd.), Cambridge, Cambridge University Press, 1994, p. 299-324.

GAZEAU, Véronique, «Recherches autour de la datation des actes normands aux $\mathrm{X}^{\mathrm{e}}$ XIIe siècles", Dating Medieval Undated Charters, Michael GERVERS (éd.), Woodbridge, The Boydell Press-Collegium Budapest, 2000, p. 61-79.

GUYOTJEANNIN, Olivier, «L'influence pontificale sur les actes épiscopaux français (provinces ecclésiastiques de Reims, Sens et Rouen, $\mathrm{XI}^{\mathrm{e}}$-XIII ${ }^{\mathrm{e}}$ siècle », in L'Église de France et la Papauté (XI ${ }^{e}-X I I I^{e}$ siècle). Actes du XXVI e colloque historique franco-allemand (Paris, 17-19 octobre 1990), Rolf Grosse (éd.), Bonn, Bouvier, 1993, p. 83-102.

POTTS, Cassandra, «The Early Norman Charters: A New Perspective on an Old Debate», in England in the Eleventh Century, Carola HICKS (éd.), Stamford, Paul Watkins (Harlaxton Medieval Studies, II), 1992, p. 25-40. 
ROUET, Dominique, «Une dépendance de l'abbaye Saint-Pierre-de-Préaux: le prieuré Sainte-Radegonde de Neufchâtel-en-Bray d'après les sources de l'abbaye de Préaux ", Annales de Normandie, 49e année, 5, décembre 1999, p. 515-538.

ROUET, Dominique, Entre gestion et historiographie: les cartulaires monastiques de la Normandie moyenne ( $X^{e}$-XII ${ }^{e}$ siècles). L'exemple des cartulaires de Notre-Dame de Mortemer, Saint-Pierre de Préaux et Saint-Martin de Troarn, Diplôme de conservateur de bibliothèque, ENSSIB, Lyon, 2000, 102 + XLII p. (dactyl.).

ToCK, Benoît-Michel, «Les chartes originales de l'abbaye de Jumièges jusqu'en 1120 », Tabularia «Études», n² 2, 2002, p. 1-19.

\section{Des sources démultipliées}

De la «mutation documentaire » à la « révolution documentaire» (J. Le Goff), il n'y a qu'un pas que nous franchirons pour souligner l'élargissement de l'éventail des sources dont disposent aujourd'hui les historiens de la Normandie ducale. À la faveur d'une relance de l'histoire rurale dans les années 1990, l'exploration de la documentation écrite a suscité un effort d'inventaire, de description, de datation, de typologie, d'édition et de critique des textes dont ont bénéficié des séries documentaires (censiers, terriers, comptabilités...) qui n’avaient pas reçu toute la sollicitude voulue ${ }^{28}$.

ANGERS, Denise, BebeAR, Catherine, DuboIs, Henri, Un censier normand du XIII ${ }^{e}$ siècle. Le Livre des Jurés de l'abbaye de Saint-Ouen de Rouen, Paris, CNRS Éditions (Documents, études et répertoires publiés par l'IRHT, 62), 2001, 478 p.

BAUDUIN, Pierre, «Trois cas de peuplement en franchises en Normandie orientale: Villedieu-la-Montagne, Gourchelles, Criquiers (1168-1306)», Histoire et sociétés rurales, $\mathrm{n}^{\circ} 15,1^{\text {er }}$ semestre 2001, p. 131-164.

WALMSLEY, John, «Les revenus de l'abbaye Saint-Amand de Rouen. Un rouleau de "rentes" des années 1220-1240», Histoire et Sociétés Rurales, n 13, 2000, p. 143-174.

ANGERS, Denise, «Terriers et livres-terriers en Normandie (XIII ${ }^{e}-\mathrm{XV}^{\mathrm{e}}$ siècle) », in Terriers et plans-terriers du XIII a a XVIII siècle. Actes du Colloque de Paris (23-25 septembre 1998), Ghislain BRUNEL, Olivier GUYOTJEANNIN, Jean-Marc MoricEAU (éd.), Rennes-Paris, Association d'Histoire des Sociétés Rurales-Ecole Nationale des Chartes (Bibliothèque d'Histoire Rurale, 5 - Mémoires et Documents de l'École des Chartes, 62), 2002, p. 19-35.

JARRY, Thomas, Terriers et Plans parcellaires de Basse-Normandie (XIII ${ }^{e}$-XVIII e siècle), Enquêtes Rurales, 5, Caen (Cahiers de la Maison de la Recherche en Sciences humaines, XVII), 1998, $131 \mathrm{p}$.

28. ARNOUX, Mathieu, BRUNEL Ghislain, «Réflexions sur les sources médiévales de l’histoire des campagnes. De l'intérêt de publier les sources, de les critiquer et de les lire», Histoire et Sociétés Rurales, 1, $1^{\text {er }}$ semestre 1994, p. 11-35, et plus particulièrement p. 25-28 («Les censiers normands: une série à constituer »). 
Les travaux récents sur les rôles de l'Échiquier de Normandie ont montré la transformation radicale de l'administration fiscale dans le duché au cours des années 1190 et souligné l'intérêt de cette source pour notre connaissance des villes, des échanges, de l'inflation monétaire, de l'organisation de la guerre et, de manière plus générale, des structures administratives de l'État normand. L'absence d'une édition moderne des rôles, les erreurs ou les lacunes des anciennes et l'utilisation parfois erronée qui a pu en être faite ont rendu indispensable une nouvelle édition mise en œuvre à l'Université de Reading par Vincent Moss. Ce travail, qui sollicite les ressources de l'informatique, sera en même temps un instrument pour l'étude de la société et de l'État normands à la charnière des XII ${ }^{\mathrm{e}}-\mathrm{XIII}{ }^{\mathrm{e}}$ siècles, en permettant par exemple d'expliquer le fonctionnement de l'Échiquier, de faire un état de la géographie administrative du duché, de stimuler la recherche en matière d'histoire économique et sociale, d'identifier les quelque 8000 personnages mentionnés dans les rôles et d'offrir ainsi des matériaux indispensables à l'analyse scientifique de la classe dirigeante et administrative du duché. Sur ce point, il devrait compléter utilement la base de données prosopographique et documentaire élaborée par Katharine KeatsRohan (COEL : Continental Origins of English Landholders, 1066-1166) à partir de 95000 noms recensés dans le Domesday Book, les Pipes rolls et près de 5000 chartes anglaises.

Moss, Vincent, «Normandy and England in 1180 : The Pipe Roll Evidence ", in England and Normandy in the Middle Ages, David BATES et Ann CURRY (dir.), Londres. Rio Grande, The Hambledon Press, 1994, p. 185-195.

Moss, Vincent, «The Norman Fiscal Revolution, 1193-98», in Crises, Revolution and Self Sustained Growth, M. OMrod, M. et R. BONNEY (éd.), Oxford, 1999, p. 3857.

Moss, Vincent, «The Norman Exchequer Rolls of King John», in King John: New Interpretations, Stephen D. CHURCH (éd.), Woodbridge, Boydell Press, 1999, p. 101116.

Ce n'est pas uniquement la diversité des matériaux qu'il faut prendre en considération, mais aussi celle des approches permettant de tirer parti tout à la fois des éléments factuels fournis par la source et de l'objet même que représente le document. Dire que tout témoignage - une charte, un cartulaire, la production d'un atelier de potier - a d'abord une réalité matérielle n'est pas faire preuve d'originalité, sauf à souligner le défi épistémologique que représente pour l'historien la maîtrise d'outils techniques de plus en plus performants et la critique de sources dont il n'est pas forcément familier. De ce point de vue l'un des apports du colloque de Cerisy sur la Tapisserie de Bayeux a été de démontrer la synergie possible des savoirs techniques relatifs à l'objet et les interprétations élaborées par les historiens, les spécialistes de l'art ou des langues. La remarque vaut également pour le traitement d'autres sources iconographiques comme l'enluminure ou l'étude des inscriptions médiévales sur tous supports, qui devrait bénéficier de la parution du volume du Corpus des inscriptions de la France médiévale consacré à la Normandie. 
Corpus des inscriptions de la France médiévale, 22, Calvados, Eure, Manche, Orne, Seine-Maritime, textes établis et présentés par Robert FAVREAU et Jean Michaud $(\dagger)$, Paris, CNRS Éditions, 2003, 500 p.

Manuscrits et enluminures dans le monde normand ( $X^{e}-X V^{e}$ siècle), Actes du colloque de Cerisy-la-Salle, octobre 1995, Pierre BoueT et Monique DosDAT (éd.), Caen, Presses Universitaires de Caen, 1999, 227 p.

La Tapisserie de Bayeux: l'art de broder l'histoire, Actes du colloque de Cerisy-la-Salle, octobre 1999, Pierre Bouet, Brian Levy et François Neveux (éd.), Caen, PUC (à paraître).

GAMESON, Richard, The Manuscripts of Early Norman England (c. 1066-1130), Oxford, Oxford University Press, 1999, XVII + 190 p.

L'intérêt du décloisonnement des champs disciplinaires n'est plus à démontrer. Les ressources de la linguistique - en particulier dans les domaines de la dialectologie, de l'anthroponymie et de la toponymie - ont été abondamment sollicitées pour tenter d'élucider les modalités de l'implantation scandinave en Normandie, pour souligner l'apparent paradoxe entre une présence bien attestée par la toponymie et une absence quasi totale de témoignages archéologiques qui en font foi ${ }^{29}$, pour situer la colonisation nordique de la province de Rouen dans les échanges complexes de populations entre les pays de l'Europe du Nord et du Nord-Ouest. Ces questions ont été largement débattues, dans une perspective comparatiste, lors des récents colloques sur «l'héritage maritime des Vikings en Europe du Nord-Ouest » (FlottemanvilleHague, 30 septembre-3octobre 1999) et sur «les fondations scandinaves en Occident et les débuts du duché de Normandie» (Cerisy-la-Salle, 25-29 septembre 2002).

L'Héritage maritime des Vikings en Europe de l'Ouest, Actes du colloque international de la Hague (Flottemanville-Hague, 30 septembre-3octobre 1999), Elisabeth RIDEL (éd.), Caen, Presses Universitaires de Caen, 2002, 565 p.

FELLOWS-JENSEN, Gillian «Les noms de lieux d'origine scandinave et la colonisation viking de la Normandie. Examen critique de la question », Proxima Thulé, Revue d'études nordiques, vol. 1, automne 1994, p. 63-103.

La démultiplication des sources est également redevable à l’archéologie médiévale. On rappellera ici le rôle pilote joué par Michel de Boüard dans l'épanouissement de cette discipline; l'effort de diffusion de l'information au travers des notices annuelles des bilans scientifiques des services régionaux de l'archéologie, des chroniques des fouilles de la revue Archéologie médiévale ou à l'occasion de différentes rencontres. L'acquis est immense et défie toute tentative d'exhaustivité. L'archéologie monumentale a également suscité un nombre impressionnant de publications, à commencer par les deux volumes sur l'architecture normande au Moyen Âge, récemment réédités avec une mise à jour bibliographique.

29. Un aperçu récent de la question dans NISSEN JAUBERT, Anne, « Some aspects of viking research in France ", in Vikings in the West, Steffen StUMANN HANSEN, Klaus RANDSBORG (éd.), Copenhague, Munksgaard (Acta archaeologica, 71), 2000, p. 159-169, en particulier p. 165-169. 
L'architecture normande au Moyen Âge, publié sous la direction de Maylis BAYLÉ, CaenCondé-sur-Noireau, Presses Universitaires de Caen - Ch. Corlet, 1997, 2e éd. 2001, 383 et $445 \mathrm{p}$.

La confrontation des textes et de l'archéologie s'est révélée ainsi particulièrement fructueuse pour notre compréhension des lieux de pouvoirs de la Normandie ducale. L'exemple de Fécamp a été largement illustré dans les communications précédentes. Les fouilles opérées à Rouen ont suggéré une profonde réorganisation de l'espace urbain à la fin du $\mathrm{IX}^{\mathrm{e}}$ siècle, peut-être comparable aux transformations opérées à Winchester à la même époque. Cette refonte a été associée par Jacques le Maho à une mutation des fonctions de la ville, devenue lieu de refuge pour la population marchande et artisanale contrainte d'abandonner aux Scandinaves les installations portuaires dispersées le long de la basse vallée de la Seine. La cession à Rollon d'une ville revitalisée par cet apport a pu ainsi contribuer à la réussite de la greffe nordique en Neustrie, que d'autres travaux sur l'insertion des normands dans le monde franc ont examiné sous des angles différents. La ville des $\mathrm{X}^{\mathrm{e}}-\mathrm{XII}{ }^{\mathrm{e}}$ siècles se laisse également mieux saisir par les données recueillies lors des opérations de fouilles et qui ont contribué, par exemple, à sortir de l'ombre les réalisations architecturales du règne de Richard $\mathrm{I}^{\mathrm{er}}$ ou les maisons citadines. L'espace urbain rouennais est ainsi mieux compris et peut être confronté à d'autres exemples qu'ont éclairées les synthèses consacrées à Caen, et, pour une période plus tardive, Bayeux et Lisieux ${ }^{30}$.

GAUTHIEZ, Bernard, «Les maisons de Rouen, XII ${ }^{e}$ XVIII ${ }^{e}$ siècles », Archéologie médiévale, XXIII, 1993, p. 131-217.

JEAN-MARIE Laurence, Caen aux XI et XII siècles. Espace urbain, pouvoirs et société, Caen, La Mandragore, 2000, 349 p.

LE MAHO, Jacques, «Les fouilles de la cathédrale de Rouen de 1985 à 1993. Esquisse d'un premier bilan ", Archéologie Médiévale, XXIV, 1994, p. 1-49.

LE MAHO, Jacques, «Autour d'un millénaire : l'œuvre architecturale à Rouen de Richard $1^{\text {er }}$, duc de Normandie (†996) ", Bulletin des Amis des Monuments Rouennais, octobre 1995-septembre 1996, p. 62-83.

LE MAHO, Jacques, «Nouvelles hypothèses sur l'église Notre-Dame de Rouen au $\mathrm{X}^{\mathrm{e}}$ siècle», Chapitres et cathédrales en Normandie, Actes du XXXI ${ }^{e}$ Congrès des Sociétés historiques et archéologiques de Normandie, Sylvette LEMAGNEN et Philippe MANNEVILLE (éd.), Annales de Normandie, Série des Congrès des Sociétés historiques et archéologiques de Normandie, Caen, II, 1997, p. 295-306.

LE MAHO, Jacques, «Les destins comparés de deux cités de fond d'estuaire: Rouen et Nantes du VI e au Xe siècle», dans Des villes, des ports, la mer et les hommes, actes du $124^{e}$ Congrès des sociétés historiques et scientifiques, Nantes, 19-26 avril 1999, sous la direction de Philippe MANNEvilLE, Comité des Travaux Historiques et Scientifiques, 2001, p. 13-25.

30. Neveux, François, Bayeux et Lisieux Villes épiscopales de Normandie à la fin du Moyen Âge, Caen, Éditions du Lys, 1996, 703 p. 
PITTE, Dominique, «Architecture civile en pierre à Rouen du XI ${ }^{e}$ au XIII ${ }^{e}$ siècle. La maison romane», Archéologie Médiévale, XXIV, 1994, p. 251-299.

COllet, Christophe, Leroux, Pascal, Marin, Jean-Yves, Caen, cité médiévale. Bilan d'archéologie et d'histoire, Caen, SDAC, 1996, 391 p.

La société normande ne se laisse plus aborder sans prendre en considération les acquis de la castellologie. Le recensement des sites fortifiés a été poursuivi par les services départementaux et régionaux de l'archéologie, des unités de recherches comme le Centre de recherches Archéologiques et Historiques Médiévales. Il a été ainsi possible, pour certains départements, comme l'Orne, de proposer un essai d'inventaire et de classement de cette masse documentaire, de présenter un schéma de mise en place du réseau castral. Les résultats de plusieurs fouilles réalisées ou entamées dans les années 1990 sont accessibles dans diverses publications, c’est le cas pour le château de Domfront ${ }^{31}$, le «Vieux Château » de Vatteville-la-Rue ${ }^{32}$, de la motte de Rivray à Condé-sur-Huisne ${ }^{33}$, de la butte de Bretoncelles ${ }^{34}$, de ChâteauGaillard aux Andelys ${ }^{35}$. Outre l'intérêt des données recueillies pour analyser les modes de construction, la typologie des constructions castrales et les aspects matériels de la vie quotidienne mis au jour sur les sites, les recherches entreprises s'inscrivent dans les problématiques fondamentales de l'histoire politique et sociale du duché (naissance des seigneuries châtelaines, élaboration des grandes dominations territoriales sur les confins méridionaux du duché, comme la seigneurie de Bellême ${ }^{36}$ ou le comté du Perche ${ }^{37}$ ). Elles participent également à un questionnement plus vaste sur l'occupation du sol, les formes de l'habitat et l'organisation du territoire.

"Autour du château médiéval», Actes des Rencontres Historiques et Archéologiques de l'Orne, Alençon, 5 avril 1997, Bruno FAJAL (éd.), Alençon, Société historique et archéologique de l'Orne (coll. «Mémoires et documents », n 1), 1998, 252 p.

Château-Gaillard: découverte d'un patrimoine (catalogue de l'exposition, Vernon, Musée municipal A. G. Poulain, 15 novembre 1995 - 6 février 1996), Dominique PitTe, Sophie Fourny-DARgÈre, Paola CALDÉroni (éd.), Vernon, Musée municipal A. G. Poulain. 1995, 148 p.

DECAËNS, Joseph, «De la motte de conquête (XI ${ }^{e}$ siècle) à la seigneurie châtelaine (XII siècle). L'exemple de Rivray à Condé-sur-Huisne», Château-Gaillard, XVI,

31. Orne, ch.-1. cant.

32. Seine-Maritime, cant. Caudebec-en-Caux.

33. Orne, cant. de Rémalard.

34. Orne, cant. de Rémalard.

35. Eure, ch.-l. d'arrond.

36. LOUISE, Gérard, «Châteaux et frontière seigneuriale au XIe siècle : l'exemple du Saosnois aux confins de la seigneurie de Bellême et du comté du Maine », Château Gaillard, XIV, 1990, p. 225-239; id., La Seigneurie de Bellême $X^{e}$-XII ${ }^{e}$ siècles, dévolution des pouvoirs territoriaux et construction d'une seigneurie de frontière aux confins de la Normandie et du Maine à la charnière de l'An mil, Le Pays Bas-Normand, 1990 ( $\left.\mathrm{n}^{\circ} 3-4\right)$; 1991 ( $\mathrm{n}^{\circ} 1-2$ ), Flers, 1992-1993, 2 vol., 432 et 351 p.

37. Thompson, Kathleen, Power and Border Lordship in Medieval France. The County of the Perche, 10001226, Woodbridge, Boydell Press, 2002, XI + 225 p. 
actes du colloque international tenu à Luxembourg (Luxembourg) du 23 au 29 août 1992, Caen, Publications du CRAM, 1994, p. 109-120.

DECAËNS, Joseph, «Les Châteaux de la vallée de l'Huisne dans le Perche», AngloNorman Studies, XVII, 1995, p. 3-20.

DECAËNS, Joseph, «La motte comme moyen de conquête du sol et comme instrument de la seigneurie châtelaine (XI ${ }^{e}-X I I I^{e} s$. $)$ : l'exemple de quelques châteaux à motte du Perche", in Aux sources de la gestion publique, t. III, Hommes de Pouvoirs, Ressources et lieux de Pouvoir ( $V^{e}$-XIII ${ }^{e}$ s.), Actes du colloque du 26 et 27 janvier 1997, Elisabeth MAGNOU-NORTIER (éd.), Lille, PUL, Septentrion, 1997, p. 263-281.

Flambard HERICHER, Anne-Marie, «La construction dans la basse vallée de la Seine: l'exemple du château de Vatteville-la-Rue (Seine-Maritime) », Château-Gaillard, XVIII, Caen, Publications du CRAM, 1998, p. 93-102.

Flambard Hericher, Anne-Marie, Bernouis, Philippe, DeCAËNS, Joseph, «La «Butte du Château » à Bretoncelles. Un exemple de la conquête territoriale des Rotrou », Château-Gaillard XIX, actes du colloque international de Graz (Autriche) 22-29 août 1998, Caen, Publications du CRAM, 2000, p. 75-82.

Flambard Hericher, Anne-Marie, «Le château des comtes de Meulan à Vattevillela-Rue, étude comparative d'une demeure aristocratique normande». Actes du colloque Aux marches du palais, Qu'est-ce qu'un palais médiéval? VII ${ }^{e}$ congrès international d'archéologie médiévale, Le Mans-Mayenne 9, 10 et 11 sept. 1999, Publications du LHAM, Université du Maine, 2001, Le Mans, p. 213-221.

Flambard Hericher, Anne-Marie, «Méthodes de recherche archéologique sur les châteaux de terre et de bois. L'exemple de la Normandie ", ESTMA IV, IV European Symposium for teachers of medieval archaeology, Séville-Cordoue, 29th september$2^{\text {nd }}$ october 1999, Séville, 2001, p. 99-112.

Flambard Hericher, Anne-Marie, «Fortifications de terre et résidence en Normandie », Château Gaillard XX, Actes du 20 colloque international « Château Gaillard », tenu à Gwatt (Suisse) 2-10 septembre 2000, Caen, Publications du CRAHM, 2002, p. $87-100$.

FlAMBARD HERICHER, Anne-Marie, «Quelques réflexions sur le mode de construction des mottes en Normandie et sur ses marges ", in Mélanges Pierre Bouet, Cahier des Annales de Normandie, $n^{\circ} 32$, Caen, 2002, p. 123-132.

FLORI, Jean, «Châteaux et forteresses aux XI ${ }^{e}$ et XII ${ }^{e}$ siècles. Étude sur le vocabulaire des historiens des ducs de Normandie", Le Moyen Âge, CIII, 2, 1997, p. 261-273.

LE MAHO, Jacques, «Fortifications de siège et «contre-châteaux» en Normandie (XIeXII ${ }^{e}$ s.) ", Château-Gaillard XIX, actes du colloque international de Graz (Autriche) 22-29 août 1998, Caen, Publications du CRAM, 2000, p. 181-189.

LE MAHO, Jacques, «L'enceinte fortifiée de Notre-Dame de Gravechon (Seine-Maritime) XI ${ }^{-}-\mathrm{XIII}{ }^{\mathrm{e}}$ siècle», Ville de Notre-Dame de Gravenchon/Groupe de recherche Archéologique du Pays de Caux, s.l., 2001, 64 p.

LOUISE, Gérard, "Châteaux et pouvoirs dans le Domfrontais médiéval (XI ${ }^{\mathrm{e}}$ XIII ${ }^{e}$ siècle) ", Les conférences d'histoire locale du lycée de Domfront, XIII, 9, 1993, p. 11-28.

MESQUI, Jean, «Le château de Gisors aux XII et XIII" siècle», Archéologie médiévale, XX, 1990, p. 253-317. 
PITTE, Dominique, «Le vieux château de Vernon : XII ${ }^{e}$ XVIII ${ }^{e}$ : : une approche historique et archéologique», Vernon, Musée A.-G. Poulain, 1990, 42 p.

PITTE, Dominique, «Château-Gaillard et la défense de la Normandie orientale (11961204)", Anglo-Norman Studies, XXIV (2001), p. 163-175.

RENOUX, Annie, Fécamp, du palais ducal au palais de Dieu, Bilan historique et archéologique des recherches menées sur le site du château des ducs de Normandie, $I I^{e}$ siècle A.C.-XVIII siècle P.C., Paris, C.N.R.S, 1991, 734 p.

RENOUX, Annie, «Palais capétiens et normands à la fin du X $X^{e}$ siècle et au début du XIe siècle", in Le Roi de France et son royaume autour de l'an Mil, actes du colloque Hugues Capet 987-1987, la France de l'an Mil, Paris-Senlis 22-25 juin 1987, études réunies par Michel PARISSE et Xavier BARRAL I ALTET, Paris, Picard, 1992, p. 179-191.

Ces thèmes sont également au cœur des débats qui ont lieu sur la genèse du village médiéval dont l'approche a été largement renouvelée par les archéologues. Les années 1990 ont vu l'achèvement des opérations archéologiques conduites aux alentours de Caen sur les sites de Saint-Martin de Trainecourt ${ }^{38}$ et de la Sente ${ }^{39}$, la reprise des fouilles du village déserté de Saint-Ursin-de-Courtisigny ${ }^{40}$, auxquels s'ajoutent les fouilles menées à Giberville ${ }^{41}$, à Vieux-Fumé ${ }^{42}$, à Tournedos-sur-Seine ${ }^{43}$, à Bouafles $^{44}$...

CARPENTIER, Vincent, «Un cas d'occupation du sol à l'époque carolingienne: le site du «Mesnil », à Plomb », Revue de l'Avranchin et du Pays de Granville, t. 77, nº 384 (septembre 2000), p. 177-187.

CARPENTIER, Vincent, «Un habitat des XI ${ }^{e}$-XII ${ }^{e}$ siècles dans la campagne d'Argentan (Orne)», Archéologie médiévale, XXXII, 2002, p. 69-103.

BURNS, Robert, BATT, Michael, «Un habitat côtier du XII siècle. Le port aux Malades, Guernesey (îles Anglo-Normandes) », Revue archéologique de l'Ouest, 7, 1990, p. 111114.

Cent maisons médiévales en France (du XII e au XVI e siècle). Un corpus et une esquisse, Jean EsQuieu et Jean-Marie PESEZ (dir.), Paris, CNRS éditions (Monographie du CRA, 20), 1998 (Grentheville, Claude Lorren, p. 205-206; Rouen, Dominique Pitte et Yves Lescoart, p. 207-212).

HANUSSE, Claire, «L'habitat rural du haut Moyen Âge (VI'- $\mathrm{X}^{\mathrm{e}}$ siècle) de «la Sente » à Grentheville (Calvados) : premiers éléments de synthèse », ETSMA III, Actes du III Colloque Européen des Professeurs d'Archéologie Médiévale (Caen, 11-15 septembre 1996), Caen, Publications du CRAM, 1999, p. 85-93.

LORREN, Claude, «Le village en Gaule du Nord pendant le haut Moyen Âge. Quelques remarques et hypothèses suscitées par l'observation des résultats des fouilles

\footnotetext{
38. Calvados, cant. Caen 7, comm. Mondeville.

39. Calvados, cant. Bourguébus, comm. Grentheville.

40. Calvados, cant. Creully, comm. Courseulles-sur-Mer.

41. Calvados, cant. Troarn.

42. Calvados, cant. Bretteville-sur-Laize.

43. Eure, cant. Val-de-Reuil.

44. Eure, cant. Les Andelys.
} 
archéologiques récentes", ETSMA III, Actes du III Colloque Européen des Professeurs d'Archéologie Médiévale (Caen, 11-15 septembre 1996), Caen, Publications du CRAM, 1999, p. 116-132.

Hincker, Vincent, Maneuvrier, Christophe, San Juan, Guy, Thiron, Denis, «Des vestiges d'habitats des $\mathrm{XI}^{e}-\mathrm{XII}{ }^{e}$ et $\mathrm{XIII}^{\mathrm{e}}-\mathrm{XV}^{\mathrm{e}}$ s. sur le site de la déviation de Vieux-Fumé (Calvados) », Histoire et traditions populaires du canton de Saint-Pierre-sur-Dives, 68, décembre 1999, p. 7-18.

HINCKER, Vincent, MANEUVRIER, Christophe, SAN JUAN, Guy, «L'habitat des XI ${ }^{\mathrm{e}}$ $\mathrm{XII}^{e}$ siècles de Vieux-Fumé (Calvados)», in La maison médiévale en Normandie et en Angleterre, Actes des tables rondes de Rouen (16-17 octobre 1998) et de Norwich (16-17 avril 1999), Dominique PitTe et Brian Ayers (éd.), Rouen, Société Libre d'Emulation de la Seine-Maritime, 2002, p. 123-130.

SAINT-JORES, Jean-Xavier, HINCKER, Vincent, «Les habitats mérovingien et carolingien de la «Delle sur le Marais » à Giberville (Calvados)», Archéologie Médiévale, XXXXXXI, 2000-2001, p. 1-38.

TAuPIN, Marie-Claude, «Le hameau de Trainecourt XIII" s. - XV $^{\text {e }}$ s. », Ruralia I, Prague, 1996, p. 211-216.

Les espaces économiques sont également mieux saisis. Depuis la synthèse sur la monnaie normande présentée en 1987 par Françoise Dumas et Jacqueline PiletLemière $^{45}$, l'étude du monnayage et de la circulation monétaire a permis d'éclairer la genèse du système pluri-dénominationnel en usage et de préciser la diffusion de la monnaie anglaise en Normandie:

MOESGAARD, Jens Christian, «Trouvailles haut-normandes de deniers esterlins des XII ${ }^{e}$ XIII ${ }^{e}$ siècles ", Bulletin de la Société Française de Numismatique, 47-9, 1992, p. 441-447.

MoEsgaARd, Jens Christian, «Two Finds from Normandy of English Coins of the Norman Kings (1066-1154)», Numismatic Chronicle, 154, 1994, p. 209-213.

MoesgaARD, Jens Christian, "Coin circulation in Plantagenet North-West France (1154-1204) in the light of new evidence from Upper Normandy $»$, in Proximus, ${ }^{\circ} 2$, Actes de la table-ronde archéologique Dieppe 17 et 18 septembre 1996, 1998, p. 129135 .

L'analyse des céramiques décorées et glaçurées découvertes à Rouen et la comparaison avec d'autres lots de céramiques des $\mathrm{X}^{\mathrm{e}}-\mathrm{XI}{ }^{\mathrm{e}}$ siècles répertoriés dans l'Europe du Nord-Ouest, en particulier à York, ont confirmé l'importance des échanges entre la principauté de Rouen et les îles Britanniques dès les premiers temps de la Normandie ducale, déjà suggérés par les données anthroponymiques et toponymiques. Des sites de production de céramiques ont fait l'objet de fouilles, dont ont rendu compte plusieurs publications comme celles consacrées à l'atelier de potier de la Roche-Mabile

45. Dumas, Françoise, PILET-Lemière, Jacqueline, «La monnaie normande $\mathrm{X}^{\mathrm{e}}-\mathrm{XII}^{\mathrm{e}}$ siècle. Le point sur la recherche en 1987 ", in Les Mondes normands (VIII ${ }^{e}-X I I^{e}$ s.). Actes du deuxième congrès international d'archéologie médiévale (Caen, 2-4 octobre 1987), Henri GaLinié (éd.), Caen, Société d'archéologie médiévale, 1989, p. 125-131. 
(Orne), daté de la fin du XII e siècle, ou les actes de la table ronde organisée à Rouen en 1994 sur la céramique des XI ${ }^{\mathrm{e}}$-XVI ${ }^{\mathrm{e}}$ siècle en Normandie, Beauvaisis et Ile-deFrance. Les foyers de cette production céramique sont mieux connus, c'est en particulier le cas pour les ateliers du Molay-Littry, dont on saisit l'activité à partir du $\mathrm{XI}^{\mathrm{e}}$ siècle. Il reste encore à préciser le passage d'une structure en ateliers dispersés à de véritables centres potiers organisés tels qu'on les observe aux XIV $\mathrm{XVV}^{\mathrm{e}}$ siècles en Bessin, en Domfrontais et en Mortainais à la faveur de l'essor de l'artisanat du grès ${ }^{46}$. Ce ne sont pas uniquement les techniques, les installations, les produits et les échanges qui intéressent les chercheurs mais aussi l'organisation de cette production et des communautés qui ont contribué à leur essor. Cette problématique, où se rejoignent l'exploitation des données fournies par l'archéologie et les textes concerne également d'autres types d'activités, comme la sidérurgie, pour laquelle Mathieu Arnoux a pu souligner l'importance et la diffusion de structures de production communautaires largement ancrées dans la société rurale normande, dont elles sont incontestablement un signe de vitalité.

\begin{abstract}
ARnoux, Mathieu, Mineurs, férons et maîtres de forge. Études sur la production du fer dans la Normandie du Moyen Age, XI ${ }^{e}-X V^{e}$ s., Paris, C.T.H.S., 1993, 646 p.

La céramique du XI' au XVI siècle en Normandie, Beauvaisis, Ile-de-France, Actes de la table ronde organisée à Mont-saint-Aignan, 12 février 1994, Xavier DELESTRE, AnneMarie Flambard HÉrICHER (éd.), Mont-Saint-Aignan, Publications de l'Université de Rouen, 1995, 102 p.

Bernouis, Philippe, Dufournier, Daniel, Fajal, Bruno, «Un atelier de potier de la fin du XII siècle à la Roche-Mabile», Revue archéologique de l'Ouest, 10, 1993, p. 129139.

DUfOURNIER, Daniel, LECLER, Elisabeth, LE MAHO, Jacques, «Céramiques glaçurées et décorées des $\mathrm{X}^{\mathrm{e}}$ et $\mathrm{XI}^{\mathrm{e}}$ siècles découvertes à Rouen », Proximus, ${ }^{\circ} 2$, Actes de la table-ronde archéologique de Dieppe 17 et 18 septembre 1996, 1998, p. 151-160.

Flambard Hericher, Anne-Marie, Potiers et poteries du Bessin. Histoire et archéologie d'un artisanat rural du XIe au XX ${ }^{e}$ siècle en Normandie, Caen, Publications du CRAHM, 2002, $407 \mathrm{p}$.
\end{abstract}

Un panorama dressé à grands traits est forcément réducteur et le souhait de son auteur est qu'il devienne rapidement obsolète, à mesure que progresseront la collecte et l'étude des données relatives à la Normandie ducale. Les recherches de Véronique Gazeau ${ }^{47}$ sur les abbés bénédictins et les prolongements qui y sont envisagés sur les manuscrits et la liturgie ${ }^{48}$ sont, de ce point de vue, de bon augure. On y ajoutera

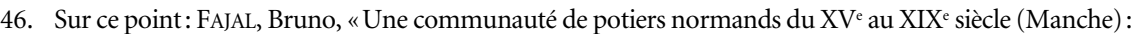
statuts et règlements du centre de Ger », Histoire et Sociétés Rurales, n 10, 1998, p. 239-263.

47. GAZEAU, Véronique, Recherches sur l'histoire de la principauté normande (911-1204), I Les abbés bénédictins de la principauté normande (911-1204), II Prosopographie des abbés bénédictins (911-1204), Mémoire d'habilitation, Université de Paris I-Panthéon-Sorbonne, 2002, 412 et 301 p. (dactyl.).

48. Sur ce point, voir également: CHADD, David, The Ordinal of the Abbey of the Holy Trinity, Fécamp (Fécamp, Musée de la Bénédictine, Ms 186), part I, Woodbridge, Boydell Press (Henry Bradshaw Society, 111), 2000, $378 \mathrm{p}$. 
l'édition des nécrologes normands, en premier lieu ceux du Mont-Saint-Michel, entreprise par Katharine Keats-Rohan. L'un des acquis les plus évidents de ces journées a été de prendre la mesure de l'effort entrepris par les historiens pour brasser des champs documentaires extrêmement différents. Gageons que la synergie déployée, relayée par les moyens de diffusion désormais offerts par les publications électroniques, trouvera là matière à de nouveaux développements. 